\title{
Le socialisme culturel et communautaire de Gustav Landauer
}

De tous les auteurs de la tradition anarchiste, Gustav Landauer (1870-1919), dont on commémore cette année le centenaire de l'assassinat, est sans doute celui qui a revendiqué le plus clairement son appartenance au socialisme, faisant de l'anarchisme une branche du socialisme et donnant même parfois les deux adjectifs « anarchiste » et « socialiste » pour de simples équivalents ${ }^{1}$. L'exposé le plus complet de ses conceptions socialistes se trouve dans l'Appel au socialisme, auquel cet article se référera principalement, sur la base d'une première traduction française à paraître ${ }^{2}$. Ces conceptions méritent aujourd'hui d'être ici revisitées, non seulement parce qu'elles sont associées à une critique du marxisme qui est redevenue audible parmi ceux qui œuvrent pour une transformation sociale radicale, mais aussi parce qu'elles cherchent à articuler expérimentations communautaires préfiguratrices et perspectives révolutionnaires.

Avant d'interroger ses possibles actualisations, nous examinerons les trois éléments qui constituent l'originalité du socialisme de Landauer. Celui-ci est d'abord un socialisme culturel, qui s'appuie sur un diagnostic historique et constate le déclin des formes de vie partagée. Il est ensuite un socialisme adossé à une critique radicale du marxisme, auquel il oppose une autre philosophie de l'histoire et une analyse sociale et politique concurrente. Il s'agit enfin d'un socialisme communautaire, qui insiste sur la nécessité de commencer ici et maintenant le socialisme sous la forme d'implantations communautaires.

Le statut de l'Appel au socialisme doit d'emblée être précisé. Il ne s'agit ni d'une réfutation du marxisme, ni d'un programme politique, ni d'une analyse du capitalisme allemand à l'époque wilhelminienne, mais d'un appel, qui vise à produire un effet sur le public, en l'occurrence qui cherche à le rallier à une conception du socialisme. La mobilisation de cette fonction du langage confère à l'écriture une dimension pragmatique qui implique non seulement une forme d'oralité qui chez Landauer peut confiner au ton prophétique - mais aussi parfois d'outrance, lorsqu'il s'agit de tordre certains bâtons, incurvés par le marxisme, dans l'autre sens.

\section{Un diagnostic culturel : le socialisme «plus mal que jamais »}

Le socialisme de Landauer s'est vu très tôt accolé le qualificatif de « culturel $^{3}$ », en raison des traits qu'il partage avec la Kulturkritik, ou critique culturelle, qui insiste moins sur les rapports d'oppression de classe inhérents au mode de production capitaliste qu'aux conséquences de ce dernier sur la culture en général. La jonction entre un idéal socialiste libertaire et ce type de critique pourrait surprendre, dans la mesure où la critique culturelle a souvent été lue comme une critique conservatrice de la modernité capitaliste et comme l'expression d'un désarroi au sein de la petite bourgeoisie menacée par la double ascension de la bourgeoisie d'affaires et du prolétariat industriel ${ }^{4}$. La critique landauerienne du capitalisme industriel partage avec ce type de critique son insistance sur le destin de l'humanité plus que sur la question ouvrière, sa remise en cause de l'idée de progrès qui va de pair avec un certain type de conservatisme ${ }^{5}$, et son idéalisme revendiqué. L'orientation culturelle du socialisme landauerien colore le diagnostic qui ouvre son Appel au socialisme, où il est d'abord question de ce qui éloigne l'époque historique qui est la sienne de toute forme de socialisme. Landauer insiste notamment sur ce qui a été perdu avec le développement du capitalisme industriel : les formes de vie partagée, mais aussi les formes de culture en commun de la

1 Sur cette équivalence, voir dans ce numéro Anatole Lucet, «La controverse entre Gustav Landauer et le Vorwärts ».

2 Gustav Landauer, Appel au socialisme, tr. fr. J.-C. Angaut et A. Lucet, La Lenteur, 2019. La pagination indiquée (dans le corps du texte pour alléger l'appareil de notes) est celle de l'édition allemande la plus récente : Gustav Landauer, Ausgewählte Schriften, éd. S. Wolf, vol. 11, Verlag Edition AV, 2015.

3 Voir par exemple Wolf Kalz, Gustav Landauer: Kultursozialist und Anarchist, Hain, 1967.

4 Pour une discussion de cette approche, voir Aurélien Berlan, La Critique culturelle et la constitution de la sociologie allemande : Ferdinand Tönnies, Georg Simmel et Max Weber, Thèse de doctorat en philosophie, Rennes, 2008.

5 Voir dans ce numéro Renaud Garcia et Anatole Lucet, « Famille et société. Réflexions sur l'anarchisme 'conservateur' de Gustav Landauer». 
terre, la généralisation du «philistinisme », jusque dans les rangs du prolétariat industriel. Mais si, pour Landauer, « le socialisme est plus mal que jamais » (p. 36) et si l'on n'a jamais été aussi loin d'une réalisation du socialisme, c'est que l'avènement du capitalisme en Europe (et le propos de Landauer ne se cantonne pas à l'irruption brusque et tardive du capitalisme industriel en Allemagne à partir du milieu du XIXe siècle, mais porte sur une phase historique qui a commencé avec la fin du moyen-âge) n'a pas seulement remis en cause un ordre social et juridique empreint d'éléments hérités de la féodalité, mais aussi la volonté de nouer librement des alliances entre êtres humains sans en passer par la médiation d'un tiers (typiquement : l'État). Cette volonté, l'auteur de l'Appel cherche à la penser à partir de la catégorie d'esprit. Or, explique Landauer dans un passage qui prend le contre-pied du marxisme, le socialisme est davantage affaire de volonté que de conscience de classe et de conditions objectives, et c'est pourquoi le socialisme est possible et impossible à tout moment et en tout lieu - possible dans la mesure où les êtres humains le veulent, impossible dans la mesure où cette volonté fait défaut. Les fluctuations de cette volonté d'alliance correspondent aux mouvements d'expansion et de contraction de l'esprit à partir de et dans des individualités d'exception, d'où la définition des génies comme « du peuple mort, du peuple vivant qui s'est concentré en eux, qui est enterré en eux et qui va renaître d'eux » (p. 41). Mais de telles individualités d'exception, sortes de concentrations de l'esprit, n'existent que parce que par ailleurs la masse est atomisée : lorsque ce n'est pas le cas, le génie est collectif. On verra que les idéaux sociaux et politiques ont le même statut.

Pour Landauer, depuis la sortie du moyen-âge, et plus encore avec l'avènement du capitalisme industriel, l'humanité occidentale connaît une période de déclin, qui pourrait aussi être une période de transition entre deux floraisons de la culture. Le révolutionnaire allemand n'hésite pas à faire l'éloge du moyen-âge chrétien, et plus généralement, non sans une certaine provocation envers le progressisme de son temps, de la stagnation comme marque de la culture. Il ne faut pas s'étonner, dès lors, de trouver dans cet Appel au socialisme, des considérations sur la primitivité à laquelle toute culture revient lorsqu'il s'agit de se renouveler, et à titre d'exemple sur la manière dont l'art byzantin a désappris la perspective. Depuis ce qu'on qualifie indûment comme Renaissance, nous sommes au contraire entrés dans « une période de déchéance parce que l'essentiel de la culture, l'esprit qui fédère les êtres humains, a décliné » (p. 112), déchéance qui peut très bien aller de pair avec le progrès des sciences et des techniques et qui se marque en tout cas par la fascination, typiquement philistine, pour ces éléments isolés de la culture. Pour l'heure, Landauer n'a pas de mots assez durs pour ce «peuple de la décadence », ce «peuple de la dégringolade », ce «peuple du déclin », « peuple qui ne peut être sauvé, ne peut être porté à la culture, que par le socialisme » (p. 46).

La dimension culturelle de la critique du capitalisme dans l'Appel au socialisme se marque aussi par son insertion dans une critique plus générale de la modernité et son association avec ces deux autres processus que sont la technique moderne et l'État moderne. Les trois éléments de ce triptyque ont en commun un même processus de dépersonnalisation et de déshumanisation : le capitalisme fait du travailleur « un numéro » (p. 107), la technique fait de lui un appendice de la machine, et les institutions étatiques automatisent la protection sociale. Chez Landauer, cette critique de la technique comme se trouvant entièrement sous l'emprise du capitalisme, et du capitalisme comme pris dans des processus techniques qu'il ne maîtrise plus, aboutit à l'affirmation suivant laquelle une nouvelle technique doit correspondre au socialisme, une nouvelle technique qui, dans le processus de travail, permettra par exemple de tenir compte des différents tempéraments, entre ceux qui travaillent vite pour se libérer du temps de loisir et ceux qui prennent plaisir à faire durer le travail. On retrouve cette coloration culturelle de la critique dans les définitions successives que Landauer fournit du socialisme dans son Appel. En tant que mouvement, le socialisme est « un effort pour créer une nouvelle réalité à partir d'un idéal », « une tendance de la volonté humaine et une vision des conditions et des voies qui mènent à l'accomplissement » (p. 36), « une tendance volontaire d'êtres humains unis pour créer du nouveau au nom d'un idéal » (p. 39), « un mouvement de culture, une lutte pour la beauté, la grandeur et la plénitude des peuples » (p. 53), « un art nouveau qui veut créer dans le vivant » (p. 151). Toutes ces définitions font primer l'élan vers un 
renouvellement de la culture sur la simple aspiration à une amélioration des conditions matérielles de vie. Le moteur du mouvement socialiste, c'est tout autant cet élan que le sentiment de vivre « des temps de décadence, d'inculture, d'absence d'esprit et de misère » (p. 37) et d'avoir à relever « des peuples déchus » (p. 39). Cette coloration culturelle peut aussi se dire dans les termes d'un primat du sentiment intérieur sur les conditions extérieures, s'il est vrai que ceux qui veulent réaliser le socialisme «souffrent avant tout intérieurement de cette situation dans laquelle ils sont pris et qui veut s'emparer d'eux jusqu'à la moelle, jusque dans leur vie, dans leur pensée, leur sentiment et leur volonté » (p. 37). Dit encore autrement, « ceux qui sont assoiffés de culture veulent créer le socialisme parce qu'ils veulent sortir du délabrement et de la misère, du capitalisme et de la pauvreté qu'il implique, de l'absence d'esprit et de la violence qui n'est que le revers de l'individualisme économique » (p. 79).

En tant qu'idéal, le socialisme n'est autre qu'une « alliance des êtres humains qui travaillent » (p. 63), « une façon bonne, pure et féconde, joyeuse, de vivre ensemble pour les êtres humains » (p. 37), une manière de conduire « des lignées humaines déclinantes vers la hauteur, la floraison, la culture, l'esprit, et pour cela vers l'alliance et la liberté » (p. 39). Landauer ne craint pas de revendiquer la dimension utopique de son socialisme. L'alternance des topies et des utopies, sorte d'équivalent des phases organiques et critiques du saint-simonisme, était déjà fortement thématisée au début de son ouvrage de 1907 sur La Révolution ${ }^{6}$. Dans l'Appel au socialisme, la dimension utopique est pensée à travers la question des rapports entre idéal et réalité. À distance d'une vision schématique qui voudrait que l'idéal devienne réalité, Landauer soutient que « c'est par l'idéal et par l'idéal seulement que notre réalité advient » (p. 37). L'utopie a donc pour fonction de faire advenir la réalité, mais celle-ci demeurera toujours plus riche que l'idéal, et celui-ci ne saurait constituer un modèle qu'il s'agirait de servilement appliquer. Cet idéal est tout autant, pour Landauer, le moteur du changement que le symptôme de la détresse dans laquelle nous nous trouvons : dans les périodes de floraison de l'esprit, il n'y a pas d'idéal, il y a une réalité riche. L'idéal, comme la grande individualité géniale, est le produit d'une réalité insatisfaisante.

La coloration culturelle du socialisme landauerien, qui le conduit à préférer le vocabulaire du peuple à celui des classes sociales, celui de la misère et de l'inculture à celui de la pauvreté, ou encore celui de la joie à celui de la conscience de classe, exclut moins la critique sociale (et les propositions politiques) qu'elle ne se la subordonne. En témoigne le fait que les classes sociales ne sont pas définies chez lui par le rapport qu'elles entretiennent à la propriété des moyens de production, mais par la conduite de vie qui est la leur : est un prolétaire celui qui vit comme un prolétaire, et qui en a les représentations intellectuelles afférentes. Chez Landauer, la critique sociale sera donc menée à partir de la question de savoir par quel type d'esprit notre travail est régulé. En l'occurrence, il s'agira plutôt de reconnaître que c'est le non-esprit (Ungeist) qui règne, et comme l'humanité a besoin de quelque chose qui tienne lieu d'esprit pour la faire tenir ensemble, se développent des succédanés, dont le principal est l'État, esprit d'un monde sans esprit, "produit de substitution pour l'esprit de peuple et de communauté qui n'existe pas » (p. 51). Cette critique sociale (ou plutôt le versant social de la critique culturelle du capitalisme moderne) est prise en charge par Landauer au travers de sa confrontation avec le marxisme.

\section{Un socialisme à rebours du capitalisme : Landauer critique du marxisme}

Il serait tentant de ne garder de la critique du marxisme par Landauer que les formules cinglantes dont il l'accable : «peste de notre temps et malédiction du mouvement socialiste » (p. 40 et 69), « superstition scientifique » (p. 54), « sens philistin érigé en système » (p. 78), « fleur de papier sur la ronce adorée du capitalisme » (p. 69), le marxisme est la cible de la partie centrale de l'Appel au socialisme. On pourrait aussi s'interroger, au-delà de la verve sardonique à laquelle Landauer donne libre cours dans ces pages, sur le type de critique qu'il produit. D'une part, celle-ci ne semble pas conserver de distinction entre Marx et le marxisme (« un rejeton qui ressemble à son père », p. 56),

6 Cet ouvrage, longtemps le seul disponible de Landauer en français, a bénéficié de deux traductions : Champ Libre, 1974 et Sulliver, 2006. 
alors que Landauer a pu, à d'autres moments de son itinéraire, opposer le Marx du Manifeste à la politique des marxistes de son temps. D'autre part, Landauer souligne lui-même que les questions que pose le courant révisionniste ou réformiste sont celles que l'anarchisme pose depuis toujours au marxisme, ce qui implique de se demander si ce qui différencie ces deux critiques du marxisme tient exclusivement aux conclusions politiques opposées qu'elles en retirent.

Mais il serait peut-être plus pertinent de revenir sur les raisons de cette place accordée à la critique du marxisme dans un texte d'appel au socialisme, qui se veut donc un texte de proposition, un texte positif, et non un texte de réfutation ou de critique. Cette place tient au moins à deux séries de raisons. En premier lieu à l'hégémonie intellectuelle du marxisme dans le socialisme allemand à l'époque où écrit Landauer. Le marxisme, dans sa variante kautskyienne, constitue en effet l'idéologie officielle du SPD (Sozialdemokratische Partei Deutschlands), qui est en passe de devenir le premier parti allemand en voix et en sièges au Reichstag, qui a vu son nombre d'adhérents dépasser le million et qui constitue le plus puissant parti socialiste européen au sein de la deuxième Internationale. Toute tentative de redéfinition du socialisme doit dès lors en passer par une confrontation avec la conception qu'en ont les marxistes. Ou pour le dire dans les termes de Landauer dans l'Appel au socialisme, il s'agit de montrer « ce que le socialisme n'est pas et ce qu'il est » (p. 54). Or dans son parcours de révolutionnaire, à l'occasion de sa participation au mouvement des socialistes indépendants qui fit suite à la révolte des Jungen au sein de l'organisation, Landauer s'est très tôt heurté à la direction du SPD, parti dont il est l'un des rares anarchistes allemands de l'époque à n'avoir jamais été membre. Dans la mesure où le marxisme, en particulier dans sa variante « orthodoxe », ne possède plus aujourd'hui cette hégémonie intellectuelle sur la critique radicale du capitalisme, on pourrait être tenté de considérer que la critique qu'en fit Landauer appartient à un stade dépassé du mouvement socialiste.

Mais en second lieu, il est également clair que cette critique du marxisme, outre qu'elle est indispensable pour quiconque écrit sur le socialisme à cette époque, est aussi l'occasion pour le révolutionnaire allemand de présenter sa propre critique sociale, ou le versant social de sa critique culturelle du capitalisme. La critique du marxisme n'est donc pas seulement affaire de conjoncture, parce qu'il s'agirait d'intervenir dans un champ dominé par le marxisme ou de détourner de ce dernier ceux qui veulent véritablement œuvrer pour le socialisme. Certaines des conceptions de Landauer - le rôle qu'il fait jouer à la volonté, à l'action en vue d'un idéal, mais aussi son attachement à des formes communautaires héritées du passé - s'opposent point par point à quelques maximes de la vulgate marxiste de l'époque, à laquelle elle paraît donc comme adossée. Mais surtout, au moment où il rédige l'Appel au socialisme, Landauer semble en être arrivé au constat que le marxisme faisait moins partie des solutions que du problème lui-même, qu'il était moins une voie qui menait au socialisme que le symptôme d'une époque où, l'esprit faisant défaut, on se rabattait sur ses travestissements. L'enjeu est donc moins d'entrer dans le détail de cette opposition ou de la caractériser par rapport à d'autres critiques du marxisme que de considérer en elle ce qui relève à proprement parler de la critique (donc des obstacles que le marxisme met en travers d'une réalisation effective du socialisme) et ce qui constitue une occasion pour Landauer de présenter sa propre critique socio-culturelle du capitalisme et sa propre conception du socialisme.

À la lecture de l'Appel au socialisme, il apparaît que trois traits fondamentaux caractérisent le marxisme pour Landauer : il est un mélange illégitime de science et d'activité partisane ; il est une mauvaise philosophie de l'histoire ; il débouche sur une philosophie de l'(in)action.

C'est autour des prétentions scientifiques du marxisme que se répartissent les premières critiques que lui adresse Landauer. Le marxisme, mixe de science et d'activité partisane, c'est « le professeur qui veut dominer » (p. 56). Son prétendu matérialisme n'est en fait qu'un économisme (partagé aussi, selon Landauer, par le syndicalisme révolutionnaire et certaines composantes de l'anarchisme) qui coupe la sphère de la production de l'économie en général, cette dernière de la vie sociale, et celle-ci de la vie de l'esprit. Landauer ne soutient pas pour autant que le socialisme serait fils de l'ignorance, mais il oppose aux prétentions scientifiques du marxisme, qui peuvent aller jusqu'à des prévisions historiques pour le moins risquées (comme Kautsky en fit régulièrement), un 
savoir vivant et délimité, qui porte à la fois sur le passé et le futur et qui pense les réalisations futures comme des renouvellements des communautés du passé.

Un deuxième ensemble de critiques tournent autour de la conception marxiste de l'histoire. Fondamentalement, et c'est le sens de la lecture critique et railleuse que propose Landauer de la fameuse conclusion du livre I du Capital sur la «tendance historique de l'accumulation capitaliste ", le révolutionnaire allemand s'oppose à l'idée suivant laquelle le socialisme pourrait être engendré par l'évolution immanente du mode de production capitaliste : «jamais le socialisme ne 'fleurira' à partir du capitalisme » (p. 75). Parmi les éléments qui fondent la confiance marxiste dans l'histoire, la croyance dans une opposition croissante entre un nombre de capitalistes toujours plus restreint et une grande masse toujours plus prolétarisée occupe une place centrale. Avec les révisionnistes, mais avec de tout autres perspectives politiques, Landauer estime cette croyance infondée : «l'état médian »s'est étoffé, et avec lui le nombre de personnes qui, sans être des capitalistes, sont personnellement intéressées à la perpétuation du capitalisme. Il insiste encore sur la manière dont le capitalisme s'est approprié des formes socialistes (la forme coopérative, par exemple) pour atténuer la concurrence entre entreprises et former une alliance des capitalistes contre les consommateurs, ainsi que sur le rôle joué par la législation sociale, non seulement pour atténuer les effets les plus brutaux du capitalisme, mais aussi pour augmenter la confiance dans l'État et affaiblir «l'humeur révolutionnaire des masses » (p. 91).

Derrière cette contestation de la foi du marxisme dans l'histoire, on trouve chez Landauer une double remise en cause (déjà présente chez Eugen Dühring) de la pensée dialectique, assimilée à un ensemble de tours de passe-passe (le plus spectaculaire étant la négation de la négation, l'expropriation des expropriateurs) qui cherchent le remède dans le mal, et du progressisme en tant qu'attitude intellectuelle consistant à fonder sur les progrès de la science et de la technique une confiance absolue dans l'avenir $^{7}$. Le marxisme illustre à merveille, pour Landauer, cette dernière attitude : «Le marxisme, c'est le philistin, et le philistin ne connaît rien de plus important, rien de plus grandiose, rien qui lui soit plus sacré que la technique et ses progrès. » (p. 73)

De ce point de vue, le marxisme apparaît à Landauer comme une sorte de philosophie de la démobilisation (« un appel à l'impuissance », p. 121), qui, en raison de sa fascination philistine pour l'évolution technique du mode de production capitaliste, prêche l'inaction au motif que les conditions objectives pour la révolution ne seraient pas réunies. Or attendre le bon moment, c'est le repousser dans le temps. Le marxisme, c'est ce courant de pensée qui estime que la révolution sociale découlera naturellement de ces trois processus qu'il tend à confondre et qui sont caractéristiques de la modernité : la centralisation des capitaux, la centralisation technique liée à l'usage de la machine à vapeur («le père du marxisme, c'est la vapeur », souligne encore Landauer, p. 73), et la centralisation politique de l'État.

Cette confiance démobilisatrice en les processus à l'œuvre dans la modernité fonde également le rôle accordé démagogiquement au prolétariat. Lorsque Landauer, qui n’a jamais renié son appartenance originelle à la bourgeoisie, dénonce dans l'Appel au socialisme le «philistinisme du prolétaire » (p. 78), il s'agit moins d'un mépris de classe ou d'une négation de toute capacité de réalisation socialiste de la part des prolétaires que de souligner que ce n'est pas en tant que prolétaires que ceux-ci pourront agir contre le capitalisme et réaliser le socialisme. Le prolétariat n'est pas « une classe révolutionnaire, mais une tapée de pauvres hères, voués à vivre et mourir au sein du capitalisme » (p. 96). Le prolétariat étant une réalité engendrée par le capitalisme, agir en prolétaire, c'est agir en conformité avec la place qui est assignée à cette classe par le régime de production capitaliste. Autrement dit, pour faire un socialiste, il faut défaire un prolétaire (ou un curé, un fonctionnaire, etc.). Les prolétaires sont de leur temps, et la transformation ne peut être initiée que par des éléments qui ne sont pas de leur temps, qui se trouvent spirituellement dans une situation d'extériorité par rapport au fonctionnement du système, qui exècrent ce dernier. Le refus d'une lecture étroitement classiste du capitalisme et de son possible renversement va, chez

7 Sur la possible déconnexion entre progressisme et socialisme, voir Dwight MacDonald, Le Socialisme sans le progrès [1946], La Lenteur, 2011. L'auteur ne semble pas connaître Landauer, qui est pourtant un contre-exemple éclatant au lien historique entre socialisme et progressisme. 
Landauer, jusqu'à une reconnaissance des tourments qu'endure le capitaliste, qui doit concilier les exigences de son dispositif d'hommes et de machines d'une part et les fluctuations des prix sur le marché mondial d'autre part.

Le refus d'assigner une mission historique rédemptrice à une catégorie déterminée par sa place dans le processus capitaliste de production nous introduit aux spécificités de la critique sociale portée par Landauer dans l'Appel au socialisme. Pour lui, il est tout à fait naturel que dans le capitalisme, les ouvriers se battent pour un meilleur partage de la survaleur. Il est en revanche illégitime de faire passer cette lutte pour une contestation du capitalisme, d'autant moins que cela revient à se battre contre les consommateurs, c'est-à-dire, s'agissant des ouvriers, contre soi-même (puisque toute augmentation des salaires s'accompagne d'un renchérissement des produits du travail). La première caractéristique du socialisme de Landauer, c'est que, contre la focalisation marxiste sur la sphère de la production, il redonne un rôle à la consommation. Pour le révolutionnaire allemand, c'est l'union d'un fort mouvement syndical pour l'augmentation des salaires et d'un puissant mouvement de coopératives de consommation qui peut conduire à une crise révolutionnaire. Ce n'est donc pas l'union des prolétaires en tant que producteurs qui pourra seule améliorer leur condition et il est nécessaire qu'ils s'unissent aussi en tant que consommateurs (dans des coopératives), sans quoi ils sont voués à se voler eux-mêmes. Autrement dit, en cantonnant la lutte sociale à la sphère de la production, en toisant avec mépris les tentatives de regroupements coopératifs, le marxisme contribue à ne pas faire sortir les travailleurs du cercle du capitalisme. Cela n'empêche pas, par ailleurs, Landauer de faire l'éloge de la lutte des syndicats pour la réduction du temps de travail, tout en rappelant que celle-ci, pour ne pas signifier une réduction de salaire, doit s'accompagner de la revendication du salaire journalier contre le salaire horaire.

Le premier enjeu du socialisme, c'est donc « le rétablissement de la relation entre travail et consommation » (p. 149). Il s'agit d'arrêter de produire pour l'argent et au contraire de se remettre à produire pour satisfaire les besoins, ce qui redonnerait son sens au travail. Pour Landauer, tous les socialistes se rejoignent pour dire que c'est le travail qui crée la valeur. La thèse suivant laquelle « $i l$ n'y a pas de productivité de la propriété et de productivité du capital, mais seulement une productivité du travail » (soulignée par l'auteur, p. 135) constitue pour lui « la connaissance fondamentale du socialisme », et c'est seulement parce qu'il l'affirme que le marxisme fait partie du socialisme. Néanmoins, l'un des torts du marxisme est d'avoir considéré que le secret de la survaleur se trouvait dans la sphère de la production - et conséquemment que la solution se trouvait aussi dans l'évolution immanente de cette dernière. Pour Landauer, si la survaleur est bien l'un des trois «pivots de l'esclavage économique » (p. 130, avec l'argent et la propriété privée du sol), elle ne naît pas spécifiquement dans la sphère de la production mais dans l'ensemble de la circulation, et sa cause est moins le travail que la contrainte au travail, ou la nécessité de travailler.

Or cette dernière naît d'une expropriation première : celle de la terre. Après le rétablissement du lien entre travail et consommation, y compris du point de vue de l'action, le rapport à la terre constitue la deuxième proposition positive du socialisme de Landauer. Dans un mouvement, auquel contribuent d'autres économistes de l'époque, dont Silvio Gesell, de revalorisation de la pensée des physiocrates, Landauer estime que si le travail est la source de toute valeur, la terre est l'origine de toute richesse. Tout en faisant grief au marxisme, dans l'Appel au socialisme, de sa focalisation indue sur la sphère de la production, Landauer n'hésite pas à concentrer ses analyses et ses propositions sur la terre et sur la possession de la terre comme point archimédien pour transformer tout le reste, en revendiquant l'abolition de la propriété de la terre au profit d'une possession qui peut être privée, coopérative ou communale. Approfondissant une critique qui est aussi ancienne que la tradition anarchiste (l'impasse sur la question paysanne), Landauer reproche au marxisme d'avoir « considéré le sol comme une forme accessoire du capital » (p. 144). De fait, à travers son concept de capital, le marxisme mélange d'un côté la terre et ses produits (tout ce qui existe effectivement), et de l'autre tout ce qui est relation, mouvement, circulation, etc., et que Landauer appelle l'effectivité économique de l'esprit fédérateur. De sorte que ce qu'on appelle capitalisme repose en fait sur du « faux capital » (p. 134 et p. 145), sur un néant qu'on prend pour une chose (au 
même titre que l'État). L'esprit, c'est le capital des capitaux. De sorte aussi que le slogan du socialisme (en écho au slogan zapatiste «Terre et liberté $\left.{ }^{8} »\right)$ doit être : «Terre et esprit » (p. 146). Pour Landauer, « les socialistes ne couperont pas à la lutte contre la propriété du sol. La lutte du socialisme est une lutte pour le sol ; la question sociale est une question agraire. » (p. 147). L'argent constitue le troisième pivot de l'oppression capitaliste. Les passages de l'Appel au socialisme sur l'argent sont fortement tributaires des analyses, mais aussi des propositions formulées par l'économiste Silvio Gesell, dont Landauer était proche et qu'il fit d'ailleurs venir à Munich au printemps 1919 au moment de la révolution en Bavière pour y occuper les fonctions de commissaire du peuple aux finances. Pour Gesell comme pour Landauer, l'argent ne peut tenir ce rôle de pivot que parce qu'il est la seule marchandise qui ne perde pas de valeur lorsqu'elle ne circule pas - d'où l'idée d'une monnaie fondante qui favorise la circulation et empêcherait thésaurisation et spéculation ${ }^{9}$. Dans le socialisme tel que le conçoit Landauer, il y aurait donc bien de l'échange, un marché, et un équivalent de l'argent.

Ces considérations socio-économiques débouchent dans l'Appel sur une série de définitions spécifiquement économiques du socialisme. Ainsi, « la forme fondamentale de la culture socialiste est l'alliance des communes économiques produisant d'une manière autonome et échangeant entre elles. » Et s'il est question de socialisme, c'est qu'il s'agit de faire advenir la société, une société qui soit « société de sociétés de sociétés », " alliance d'alliances d'alliances », «être commun de communautés de communes », « république de républiques de républiques » (p. 138), par où l'on conçoit que le processus (plus que le modèle) au travers duquel doit se réaliser le socialisme est, conformément à ce que soutient la tradition anarchiste depuis Proudhon, un processus fédératif.

\section{Communauté et révolution ${ }^{10}$}

L'un des points d'aboutissement de la critique landauerienne du marxisme est son attaque contre la « peur puérile de l'échec » qui empêche les marxistes d'agir tant que les prétendues « conditions objectives » de la révolution sociale ne sont pas réunies. Or pour Landauer, « qui ne prend pas le risque de l'échec, de l'isolement, du revers, ne parviendra jamais à la victoire » (p. 71). Cette attaque se comprend à partir de l'insistance de Landauer sur le thème de la communauté, insistance qui possède deux versants : elle est à la fois revalorisation de formes communautaires héritées et incitation à commencer tout de suite, au travers d'implantations communautaires (ou de « colonies », Siedlungen), à réaliser le socialisme, puisque « ce que les êtres humains ne font pas maintenant, ne commencent pas à faire sur le champ, ils ne le feront pas de toute éternité »(p. 156). L'Appel au socialisme se veut en effet le manifeste de l'Alliance socialiste (Sozialistischer Bund) constituée en 1908 par Landauer et qui vise à fédérer des expériences communautaires. Il faut ici rappeler l'implication personnelle du révolutionnaire allemand dans une pluralité d'initiatives locales (coopératives de consommation, projets de cités-jardins, communautés de vie) qui ne se cantonneront jamais chez lui à un « anarchisme du mode de vie ${ }^{11}$ » et auront toujours une signification sociale et politique.

Appeler au socialisme, c'est appeler à le réaliser ici et maintenant - ni dans un autre pays (comme le pensent certains utopistes de l'époque, comme Theodor Hertzka dans Freiland, ou une partie du

8 Voir Gustav Landauer, «Sur la révolution mexicaine » [1911-1914], trad. J.-C. Angaut, Réfractions, $\mathrm{n}^{\circ}$ 36, printemps 2016, p. 151-162.

9 Les idées de Gesell sur la monnaie sont synthétisées dans son livre de 1916 L'Ordre économique naturel. On notera la proximité entre certains passages de l'Appel au socialisme de Landauer (notamment p. 149, l'idée suivant laquelle l'argent est riche de ce dont nous sommes pauvres) avec le petit essai de Moses Hess Sur l'essence de l'argent, qui fut l'une des sources des Manuscrits de 1844 de Marx (publiés pour la première fois en 1927) et qui pourrait contribuer à expliquer la ressemblance par endroits de l'Appel avec un texte que Landauer ne pouvait pas connaître.

10 Je reprends ici le titre de la thèse de doctorat d'Anatole Lucet, Communauté et Révolution chez Gustav Landauer, ENS de Lyon, 2018.

11 Ce qui permet de contester l'opposition décelée par Murray Bookchin dans son essai de 1990, Social Anarchism or Lifestyle Anarchism: An Unbridgeable Chiasm. 
mouvement sioniste), ni dans un futur indéterminé (comme le suggèrent les marxistes) - et cela implique que le socialisme soit «possible à toute époque et avec toute technique » et «impossible à toute époque et avec toute technique » (p. 84). Reconnaître cette possibilité et cette impossibilité est l'un des enjeux de l'insistance de Landauer sur l'activité de Proudhon lors de la révolution de 1848. Pour Landauer, «son socialisme des années 1848-1851 était le socialisme du peuple français dans les années 1848-1851 » (p. 120), de sorte qu'en l'accusant d'être un socialiste petit-bourgeois, les marxistes rendent involontairement hommage à celui que Landauer a coutume d'appeler « le plus grand de tous les socialistes » (p. 69 et p. 83).

Adapté au présent, le projet socialiste doit aussi être ancré dans le passé. En réalisant, les socialistes doivent penser les générations à venir comme étant leurs successeurs, de même qu'ils doivent se regarder comme les héritiers des formes communautaires du passé. Si le présent est insatisfaisant, c'est qu'il a substitué à « des communes, des confédérations, des guildes, des fraternités, des corporations, des sociétés, [qui] toutes se stratifiaient pour former la société », ces éléments typiques de la modernité que sont « la contrainte, la lettre, l'État» (p. 51), et qui s'opposent terme à terme à la liberté, à l'esprit et à la société. Pour Landauer, « la vraie société est une multiplicité de petites appartenances communes effectives, qui sont nées des propriétés fédératrices des individus, qui sont nées de l'esprit » (souligné par l'auteur, p. 81). Cette inscription dans une tradition communautaire n'est pas seulement une vue de l'esprit, elle implique aussi une stratégie d'implantation. Landauer insiste ainsi sur la nécessité de s'appuyer sur « des communautés villageoises avec des restes de possession communale » et « des institutions d'économie commune pour le travail aux champs et l'artisanat » (p. 150). Conformément au modèle théorique de la régénération auquel le socialisme culturel de Landauer se rallie, mais en opposition avec toute tentation réactionnaire de restauration de l'ancien, « la nouvelle société que nous voulons préparer, dont nous nous apprêtons à poser la première pierre, ne sera pas un retour à quelques anciennes formations, elle sera l'ancien sous une nouvelle figure, elle sera une culture avec les moyens de la civilisation qui a nouvellement grandi en ces siècles. » (p. 116)

Le socialisme doit donc mettre immédiatement en œuvre une sortie du capitalisme, d'abord au moyen de ces petits commencements que sont les « colonies dans lesquelles les socialistes se coupent autant que possible du marché capitaliste et n'exportent qu'autant de valeur qu'ils doivent en recevoir de l'extérieur » (p. 152). Dans cette perspective, la communauté est avant tout un essai de sortie, qui comme tout essai est voué à être repris, amélioré, donné en exemple (sans être pour autant exemplaire, si l'on entend par là que l'expérience devrait être servilement reproduite). Pour une part, même si Landauer ne considère pas ces expérimentations comme l'alpha et l'oméga du changement social, il y a un là un modèle de transformation par prolifération : il s'agit que partout des colonies socialistes surgissent au milieu de la bassesse de l'économie du profit, donnent à voir une manière joyeuse et fraternelle de produire et de consommer et se rendent ainsi désirables. Il ne saurait être question d'émancipation « que pour ceux qui se mettent intérieurement et extérieurement en état de sortir du capitalisme, qui cessent de jouer un rôle et commencent à être des êtres humains. » (p. 137)

Un tel modèle de changement social exclut-il la révolution ? Depuis le livre qu'il a consacré à cette notion en 1907, Landauer ne cesse de répéter que celle-ci recouvre deux choses : un processus historique, enclenché dans le monde occidental depuis la Renaissance (et dont ont participé la Réforme et la Révolution française), et un processus ponctuel qui consiste dans une confrontation violente avec l'État. En ce second sens, la question révolutionnaire ne manquera pas de se poser dès lors qu'une composante importante de la population aura fait sécession, se sera retirée de l'économie du profit. Il faudra bien alors se confronter aux tentatives de l'État pour ramener la population au bercail propriétaire. Ce moment de confrontation sera un moment révolutionnaire, qui engendrera des révolutionnaires - le tort serait de croire, sur un mode éventuellement insurrectionnaliste, que ce sont les révolutionnaires qui font les révolutions, alors que c'est l'inverse : «Il n'y a de révolutionnaires en masse que lorsqu'il y a une révolution ; l'une des plus graves erreurs des marxistes, qu'ils s'appellent sociaux-démocrates ou anarchistes, est l'opinion selon laquelle on pourrait passer par des révolutionnaires pour obtenir une révolution, alors 
qu'inversement, ce n'est qu'en en passant par la révolution qu'on obtient des révolutionnaires. » (p. 92) Or le défaut des prétendus révolutionnaires, c'est qu'ils anticipent les bâtons que l'État leur mettra dans les roues et en tirent argument pour attendre que les conditions soient mûres. La révolution, ce n'est rien d'autre que la destruction des obstacles, mais lorsque ces obstacles sont réellement rencontrés dans la réalité, et pour cela, il faut qu'il y ait un mouvement de construction du socialisme réellement à l'œuvre. Autrement dit, la révolution, au sens du moment insurrectionnel, n'a de caractère effectivement révolutionnaire que si elle est adossée à une transformation sociale déjà en cours.

\section{Actualité d'un inactuel}

Il y a quelque chose de tentant à affirmer l'actualité de la pensée de Landauer, mais aussi quelque chose de délicat s'agissant d'une œuvre de plus d'un siècle. L'entreprise qui consisterait à démêler chez lui le bon grain de l'ivraie ne serait toutefois guère plus satisfaisante. On ne manquera certes pas de se détourner des pages de Landauer dans lesquelles il évoque la dégénération humaine provoquée par le capitalisme avec des accents biologisants, ni de souligner ce que sa critique du marxisme a d'intriqué à la réalité du marxisme de son époque. Mais l'essentiel est sans doute de se demander ce qui, dans cet Appel au socialisme qui est sans conteste l'un des plus beaux textes de la littérature socialiste de l'époque, peut encore constituer pour le présent une source d'inspiration. De ce point de vue, les pages de ce livre qui insistent sur l'inactualité du socialisme - non seulement au sens de ce qui n'est pas là, mais aussi de ce qui va à rebours des tendances dominantes de l'actualité - fournissent une clé paradoxale pour l'actualisation de cette pensée. Toute pensée de transformation radicale possède une composante d'inactualité, fait valoir les droits d'un passé défiguré et d'un avenir inconnu contre un présent exécrable.

Au-delà des qualificatifs qu'on a pu accoler au socialisme de Landauer (culturel, communautaire, anti-marxiste), on peut en retenir trois autres caractéristiques qui ont une pertinence pour aujourd'hui. Tout d'abord, il s'agit d'un socialisme à la fois expérimental et révolutionnaire. À l'heure d'un regain d'intérêt pour les formes communautaires, par exemple dans le cadre de résistances à de grands projets inutiles (ZAD), mais aussi pour la forme coopérative (ainsi de la Coopérative intégrale catalane, qui, en tant que tentative effective de sortir du capitalisme, aurait sans doute fort intéressé Landauer), la manière qu'a le révolutionnaire allemand de penser l'articulation entre de telles tentatives de sortie du capitalisme et un renversement révolutionnaire du capitalisme mérite toute notre attention.

En second lieu, les passages de l'Appel au socialisme dans lesquels Landauer insiste sur la nécessité de rétablir le lien perdu entre le travail et les besoins ne peuvent que nous frapper, notamment en ce qu'ils articulent quelque chose comme un souci éthique de simplicité volontaire, consistant à discerner parmi nos besoins ceux qui sont nécessaires et ceux qui sont engendrés par l'économie du profit (p. 99), et une perspective révolutionnaire qui tire les conséquences politiques et sociales de ce souci éthique du côté d'une sortie en masse hors du capitalisme.

Mais ce sont peut-être les dernières pages de l'Appel qui entrent le plus en résonance avec notre présent, en tant qu'elles mêlent le désespoir le plus profond à l'enthousiasme le plus fou. Le socialisme de Landauer, on l'a dit, est socialisme de la volonté, pour qui, faute d'un véritable enthousiasme pour d'autres manières de faire et de vivre, aucune réalisation n'est possible, ce qu'on ne peut que méditer en ces temps blasés et ironiques. Mais l'insistance sur la volonté côtoie chez lui toute une thématique quasi-apocalyptique sur la fin du monde, qui n'est pas sans analogie avec notre époque où fleurissent les tentatives plus ou moins convaincantes de « collapsologie ». Le socialisme de Landauer est aussi un socialisme du désespoir. Au-delà des accents biologisants des passages sur l'éventualité que le socialisme devienne impossible en raison de la dégradation physique des corps individuels dans le capitalisme, au-delà aussi de la dramatisation d'un propos qui, à la veille du premier conflit mondial, insiste sur l'éventualité que « l'humanité commençante » (au sens de la naissance d'une société mondiale) puisse être aussi «la fin de l'humanité »(p. 125), l'Appel au socialisme se veut une incitation, en des temps inquiétants et incertains, à «nous 
immerger dans cet abîme pour y puiser le courage et la nécessité pressante dont nous avons besoin » (p. 127). Plus encore qu'en 1911, nous serions tenté de soutenir qu' " aucune époque n'a peut-être eu plus dangereusement sous les yeux que la nôtre ce qu'on se plaît à appeler la fin du monde » (p.

125). Dans ce contexte, il vaut de méditer la réponse de Landauer : « Nous ne le savons pas, et pour cette raison, nous savons que l'essai est notre tâche » (souligné par l'auteur p. 129). Dire qu'il n'y a rien à attendre de l'histoire, ce n'est pas seulement révoquer la tentation de s'en remettre à l'histoire, c'est aussi ne pas abdiquer devant le catastrophisme ambiant.

Jean-Christophe Angaut 